\title{
DESSORÇÃO DE FÓSFORO POR SILÍCIO EM SOLOS CULTIVADOS COM EUCALIPTO ${ }^{(1)}$
}

\author{
R. CARVALHO(2), A. E. FURTINI NETO(3), N. CURI(3), \\ L. A. FERNANDES ${ }^{(4)} \&$ A. C. OLIVEIRAJ R. ${ }^{(5)}$
}

\begin{abstract}
RESUMO
Com o objetivo de avaliar a dessorção de fósforo por silício nas camadas superficiais de um Latossolo Vermelho-E scuro sob cerrado e de um Cambissolo sob campo cerrado, da zona fisiográfica Campos das Vertentes (MG), foram realizados experimentos em casa de vegetação do Departamento de Ciência do Solo da Universidade Federal de Lavras (MG), no período de agosto de 1997 a maio de 1998. Cada solo foi submetido a três incubações seqüenciais: (a) $\mathrm{CaCO}_{3}+\mathrm{MgCO}_{3}$, para manter o pH em torno de 6,0; (b) fertilização básica, incluindo o fósforo em dose única, calculada para manter $0,2 \mathrm{mg} \mathrm{L}^{-1} \mathrm{de} P$ em solução; (c) seis doses de si lício $\left(\mathrm{CaSiO}_{3}\right)$, definidas com base na dose de fósforo. Plantas de Eucalyptus grandis foram cultivadas por 120 dias em vasos com capacidade para $3 \mathrm{dm}^{3}$ de solo. A produção de matéria seca e o teor de P na matéria seca foram avaliados aos 60, 90 e 120 dias do transplantio das mudas para os vasos. Ajustaram-se superfícies de resposta dessas variáveis às doses de Si e às épocas. O P dessorvido contribuiu com $15,25 \%$ do conteúdo do nutriente na matéria seca das plantas cultivadas no Cambissolo (maior teor de caulinita).
\end{abstract}

Termos de indexação: Latossolo, Cambissolo, Eucalyptus.

\section{SUMMARY: PHOSPHORUS DESORPTION BY SILICON IN SOILS CULTIVATED WITH EUCALYPTUS}

With theobjectiveto evaluatethedesorption of phosphorus by silicon in surfacelayers of a Dark-Red Latosol (Oxisol) under cerrado vegetation and Cambisol (Inceptisol), under open cerrado vegetation at Campos das Vertentes physiographical region StateM inas Gerais,

\footnotetext{
(1) Parte da Tese de Doutorado do primeiro autor, a ser apresentada ao Departamento de Ciência do Solo da U niversidade F ederal de Lavras (UFLA), Lavras (MG). Recebido para publicação em fevereiro de 1999 e aprovado em outubro de 1999.

(2) Professor do Departamento de Química, Universidade Federal de Lavras - UFLA. CEP 37200-000 Lavras (MG).

(3) Professor do Departamento de Ciência do Solo, UFLA.

(4) Professor Adjunto do Núcleo de Ciências Agrárias, Universidade Federal de Minas Gerais - UF MG. Caixa Postal 135, CEP 39404006 Montes Claros (MG).

(5) Aluno do Curso de Graduação em Agronomia, UFLA.
} 
experiments were carried out under greenhouse conditions at the Soil Science Department of the Federal University of Lavras, Minas Gerais state, Brazil, from August 1997 to May 1998. Each soil was submitted tothreesequential incubations: (a) $\mathrm{CaCO}_{3}+\mathrm{MgCO}_{3}$ to maintain the $\mathrm{pH}$ around 6.0; (b) basic fertilization, including phosphorus in one dose calculated to maintain $0.2 \mathrm{mg} \mathrm{L}^{-1}$ of $\mathrm{P}$ in soil solution; and (c) six silicon $\left(\mathrm{CaSiO}_{3}\right)$ doses defined with basis on phosphorus dose. Seedlings of E ucalyptus grandis werecultivated during 120 days in pots with $3 \mathrm{dm}^{3}$ of soil. Dry matter production and dry matter $P$ content were evaluated at 60,90 and 120 days after seedl ing transplanting to the pots. Response surfaces of these variables were adjusted as a function of $\mathrm{Si}$ doses and times. The desorbed $\mathrm{P}$ contributed with $15.25 \%$ of the content of this nutrient in dry matter of plants cultivated in theCambisol (higher content of kaol inite).

Index terms:, Oxisol , Inceptisol, Eucalyptus.

\section{INTRODUÇÃO}

O composto de silício presente na fase líquida do solo, numa faixa de pH de 4 a 9, éo monômero $\mathrm{H}_{4} \mathrm{SiO}_{4}$ (McKeague \& Cline, 1963ab). Sua retenção, comprovada por Holt \& King (1955) por meio do Si31 (radioativo), ocorre por adsorção específica às superfícies dos óxidos de $\mathrm{Fe}$, à semelhança do $\mathrm{P}$ (Hingston et al., 1972). Fósforo e silício competem entresi pel os mesmos sítios de adsorção, de maneira que o segundo pode desl ocar (dessorver) o primeiro, e vice-versa, da fase sólida para a líquida (Obihara \& Russel, 1972; Oliveira, 1984; Leite, 1997).

Os estudos básicos relatados a seguir foram testados por diversos pesquisadores sob condi ção de casa de vegetação e campo. A aplicação de escória de siderurgia ( $\sim 20 \%$ de $\left.\mathrm{CaSiO}_{3}\right)$ em solos ácidos do Havaí (EUA), cultivados com cana-de-açúcar, reduziu em até $47 \%$ a demanda por $P$, notadamente em ambientes caul iníticos (Roy et al., 1971; Silva, 1971). Analisando um Latossolo Roxo e uma Terra Roxa Estruturada adubados com termofosfato magnesiano ( $~ 11 \%$ de Si na forma de $\mathrm{MgSiO}_{3}$ ) e cultivados com arroz, Baldeon (1995) demonstrou que o ganho em $P$ pelas plantas foi devido ao somatório de dois efeitos: o poder alcalinizante do Si e a competição Si $\times$ P pel os mesmos sítios de adsorção dos sol os. Vale a pena ressaltar que o $\mathrm{Si}$, na forma de silicato de $\mathrm{Ca}$ ou de $\mathrm{Mg}$ e em doses el evadas como no trabal ho de Roy et al. (1971), foi aplicado ao solo antes da fertilização fosfatada, à semelhança da prática da calagem. Portanto, a maior disponibilidade de P pode ter ocorrido mais por influência do incremento do $\mathrm{pH}$ do que pela competição entre Si eP pel os mesmos sítios de adsorção. Além disso, os referidos trabal hos utilizaram gramíneas como plantas indicadoras, reconhecidas como acumuladoras de $\mathrm{Si}$ em seus tecidos (Marschner, 1995). N este caso, não se pode descartar também o papel nutricional do el emento, mascarando ou confundindo-se na planta com os benefícios do P dessorvido do solo pelo Si. Existe, portanto, a necessidade de isolar estes efeitos do papel direto do silício na dessorção de fósforo no solo e, se possível, dimensionar esteúltimo.
Dessa forma, o presentetrabal ho teve por objetivo avaliar, de forma indireta em plantas, a capacidade do silício, aplicado numa etapa posterior à fertilização fosfatada, em dessorver $\mathrm{P}$ de dois solos ácidos, com teores semelhantes de óxidos de $\mathrm{Fe}$ "totais" e diferentes teores de caulinita e gibbsita cultivados com eucalipto.

\section{MATERIAL E MÉTODOS}

Amostras de Latossolo Vermel ho-Escuro (LE), fasecerrado, edeCambissolo(C), fase campo cerrado, originados de rochas pelíticas pobres do município deSãoSebastião da Vitória, zona fisiográfica Campos das Vertentes (MG), sob pastagem nativa, foram col etadas na camada de $0-20 \mathrm{~cm}$ de profundidade. Os solos foram escolhidos por apresentarem expressiva representatividade territorial no País (Resende et al., 1997).

Os solos, após secagem ao ar, for am passados em peneira de $2 \mathrm{~mm}$. As análises químicas ( $\mathrm{pH}$ emágua, $\mathrm{K}, \mathrm{Ca}, \mathrm{Mg}, \mathrm{Al}, \mathrm{H}+\mathrm{Al}, \mathrm{P}$ e carbono orgânico) foram realizadas conforme EMBRAPA (1997). Os teores de óxidos de Fe livres e totais foram obtidos por meio de duas extrações sucessivas com ditionito-citratobicarbonato de sódio (Mehra \& J ackson, 1960). Os teores dos óxidos do ataque sulfúrico foram determinados conforme Vettori (1969), com modificações de EMBRAPA (1997). A mineralogia qualitativa da fração argila foi obtida por difratometria de raios- $X$, enquanto gibbsita e caulinita foram quantificadas na fração argila desferrificada, por meio de análise térmica diferencial. A mineralogia dos óxidos de ferro foi analisada na fração argila após concentração com $\mathrm{NaOH}$ 5,0 mol L-1 (Kämpf \& Schwertmann, 1982), sendo a taxa goethita/goethita + hematita quantificada com base nos difratogramas de raios$X$. As formas menos cristalinas dos óxidos de $\mathrm{Fe}$ foram dosadas pelo oxalato de amônio, segundo Schwertmann (1973), e a capacidade máxima de adsorção de P (CMAF) conforme Syers et al . (1973). 
Os experimentos foram realizados em casa de vegetação do Departamento de Ciência do Solo da Universidade Federal de Lavras, Lavras (MG). A unidadeexperimental constou de um vaso de plástico com capacidade para $3 \mathrm{dm}^{3}$, contendo $2,7 \mathrm{dm}^{3}$ de sol o passado em peneira de $2 \mathrm{~mm}$ de diâmetro. Todos os vasos foram submetidos a três incubações seqüenciais, por um período de 30 dias cada uma, sob condição de umidade equivalente a $60 \%$ do volumetotal de poros (VTP), controlada por pesagem diária.

A primeira, com a mistura $\mathrm{CaCO}_{3}+\mathrm{MgCO}_{3}$ P.A., em quantidades definidas com base em experimento prévio de incubação, objetivou manter em 4:1 a relação Ca:Mg e o pH em torno de 6,0. A segunda, idêntica para os dois sol os à exceção do $P$, constituiuse da adição de nutrientes, na forma de sais P.A, nas doses, em $\mathrm{mg} \mathrm{dm}^{-3}$, de 80 de N, 150 de K, 40 de S, 0,81 de $B, 3,6$ de $\mathrm{Mn}, 1,3$ de $\mathrm{Cu}, 1,6$ de $\mathrm{Fe}, 0,15$ de Mo e 5 de $Z n$. As doses de $P$, estipuladas por determinação da CMAF para manter $0,2 \mathrm{mg} \mathrm{L}^{-1}$ de $P$ em sol ução, foram de $225 \mathrm{mg} \mathrm{dm}^{-3}$, para o sol o LE, e de $150 \mathrm{mg} \mathrm{dm}^{-3}$, para o solo C. A terceira incubação constou de seis doses de Si na forma de $\mathrm{CaSiO}_{3}$ (28,97\% de $\mathrm{Si}$ e $13,21 \%$ de $\mathrm{Ca}$ ). Para o solo LE, as doses foram de $0,112,170,225,280$ e $340 \mathrm{mg} \mathrm{dm}^{-3}$ de Si e, para o solo C, de 0, 75, 112, 150, 187 e $225 \mathrm{mg} \mathrm{dm}^{-3}$ de Si. Em cada solo, uma das doses de Si foi numericamente idêntica à dose de $P$ aplicada na segunda incubação. As demais corresponderam, no seu extremo inferior, a $50 \%$ da dose de $\mathrm{P}$ e, no extremo superior, a 1,5 vez a dose deP. Como a fonte de Si empregada trouxe o Ca como cátion acompanhante, procedeu-se ao seu balanceamento com $\mathrm{CaCO}_{3}$ P.A nos tratamentos afins.

Os resultados da análise quími ca realizada depois de concluídas as três incubações revelaram o nivelamento a que foram submetidas todas as características do complexo sortivo, à exceção do silício (Quadro 1). Este, em sua forma solúvel em água $\left(\mathrm{H}_{4} \mathrm{SiO}_{4}\right)$, aumentou linearmente com as doses aplicadas em cada solo.

Após a incubação, foram transplantadas quatro mudas de Eucal yptus grandis, com quatro pares de fol has, deixando-se, após o desbaste, duas mudas por vaso.

O delineamento experimental utilizado foi o inteiramente casualizado, arranjado num fatorial $6 \times 3$ (seis doses de Si etrês épocas: 60, 90 e 120 dias do transplantio), com quatro repetições.

Aos 60, 90 e 120 dias do transplantio das mudas, as plantas foram col hidas, separadas em parte aérea e sistema radicular, secas em estufa (65-70ㄷ), pesadas e moídas, sendo o $\mathrm{P}$ determinado após digestão nitroperclórica, conforme Malavolta et al. (1989), e, convertido para conteúdo de P na matéria seca da parte aérea.

Concluída a análise de variância dos resultados obtidos, foram ajustadas superfícies de resposta para as variáveis experimentais (produção de matéria seca da parte aérea de plantas e conteúdo de $P$ na matéria seca), considerando as doses deSi aplicadas e as épocas de avaliação.

Quadro 1. Atributos químicos dos solos após correção da acidez, adubação básica e aplicação dos tratamentos

\begin{tabular}{|c|c|c|c|c|c|c|c|c|c|c|c|c|}
\hline \multirow{3}{*}{ Atributo } & \multicolumn{12}{|c|}{ Dose de Si aplicada (mg dm-3) } \\
\hline & \multicolumn{6}{|c|}{ Latossolo Vermelho-E scuro } & \multicolumn{6}{|c|}{ Cambissolo } \\
\hline & $\mathbf{0}$ & 112 & 170 & 225 & 280 & 340 & $\mathbf{0}$ & 75 & 112 & 150 & 187 & 225 \\
\hline pH em água & 5,7 & 5,8 & 5,7 & 5,7 & 5,8 & 5,7 & 5,4 & 5,4 & 5,3 & 5,4 & 5,5 & 5,4 \\
\hline$P_{\text {Mehlich-1 }}\left(\mathrm{mg} \mathrm{dm}^{-3}\right)$ & 6 & 6 & 6 & 6 & 7 & 7 & 11 & 11 & 11 & 11 & 11 & 11 \\
\hline$P_{\text {Resina }}\left(\mathrm{mg} \mathrm{dm}^{-3}\right)$ & 75 & 79 & 70 & 75 & 75 & 78 & 37 & 35 & 33 & 36 & 36 & 40 \\
\hline $\mathrm{K}(\mathrm{mg} \mathrm{dm}-3)$ & 156 & 172 & 188 & 190 & 180 & 184 & 168 & 168 & 174 & 184 & 174 & 172 \\
\hline $\mathrm{Ca}\left(\mathrm{cmol}_{\mathrm{c}} \mathrm{dm}^{-3}\right)$ & 4,2 & 4,5 & 4,7 & 4,3 & 4,3 & 4,2 & 2,7 & 2,7 & 3,0 & 2,9 & 2,9 & 2,9 \\
\hline $\mathrm{Mg}\left(\mathrm{cmol}_{\mathrm{c}} \mathrm{dm}^{-3}\right)$ & 0,8 & 0,9 & 0,9 & 1,3 & 1,5 & 1,3 & 0,6 & 0,7 & 0,6 & 0,7 & 0,8 & 0,7 \\
\hline $\mathrm{Al}\left(\mathrm{cmol}_{\mathrm{c}} \mathrm{dm}^{-3}\right)$ & - & - & - & - & - & - & - & - & - & - & - & - \\
\hline $\mathrm{H}+\mathrm{Al}\left(\mathrm{cmol}_{\mathrm{c}} \mathrm{dm}^{-3}\right)$ & 2,9 & 2,9 & 3,2 & 3,2 & 3,2 & 3,2 & 4,0 & 4,0 & 4,0 & 4,0 & 4,5 & 4,0 \\
\hline $\mathrm{S}\left(\mathrm{cmol}_{\mathrm{c}} \mathrm{dm}^{-3}\right)$ & 5,4 & 5,8 & 6,1 & 6,1 & 6,3 & 6,0 & 3,7 & 3,5 & 4,0 & 3,8 & 4,1 & 4,0 \\
\hline $\mathrm{t}\left(\mathrm{cmol}_{\mathrm{c}} \mathrm{dm}^{-3}\right)$ & 5,4 & 5,8 & 6,1 & 6,1 & 6,3 & 6,0 & 3,7 & 3,5 & 4,0 & 3,8 & 4,1 & 4,0 \\
\hline $\mathrm{T}\left(\mathrm{cmol}_{\mathrm{C}} \mathrm{dm}^{-3}\right)$ & 8,3 & 8,7 & 9,3 & 9,3 & 9,5 & 9,2 & 7,7 & 7,5 & 8,0 & 7,8 & 8,6 & 8,0 \\
\hline $\mathrm{m}(\%)$ & - & - & - & - & - & - & - & - & - & - & - & - \\
\hline V (\%) & 65 & 67 & 66 & 66 & 66 & 65 & 48 & 47 & 50 & 49 & 48 & 50 \\
\hline Matéria orgânica $\left(\mathrm{g} \mathrm{kg}^{-1}\right)$ & 34 & 37 & 38 & 38 & 40 & 37 & 27 & 28 & 27 & 27 & 26 & 27 \\
\hline Si solúvel em água $\left(\mathrm{mg} \mathrm{dm}^{-3}\right)$ & 28 & 41 & 48 & 49 & 56 & 63 & 18 & 24 & 27 & 28 & 29 & 34 \\
\hline
\end{tabular}




\section{RESULTADOSE DISCUSSÃO}

A dessorção de $\mathrm{P}$ por $\mathrm{Si}$ foi quantificada em plantas, deforma indireta, por meio da determinação do ganho em $P$ pela matéria seca da parte aérea (Figuras 1c e 1d) e da contribuição desta fração adicional do nutriente para a produção de matéria seca (Figuras la e 1b).

A produção de matéria seca da parte aérea (MSPA) das plantas cultivadas no LE e no C aumentou, respondendo a incrementos das doses de Si e das épocas de avaliação (sinal positivo dos termos "Si eE" do pol inômio das figuras la e 1b). No solo C (Figura $1 \mathrm{~b}$ ), a idêntica significância dos coeficientes de doses e épocas (1\%) permite assumir que, estatisticamente, neste sol o, os dois fatores não diferiram entre si quanto à importância de influenciar a produção da MSPA. Ao contrário, no
LE, pode-se admitir que houve, embora discreta, diferença estatística mais favorável à época (1\%) que à dose. Esse fato deve ser analisado com cuidado, à luz do papel desempenhado pelo Si nas plantas. E m primeiro lugar, o el emento não é considerado essencial, mas útil aos vegetais, notadamente gramíneas (Marschner, 1995). Nestas, admite-seuma função metabólica (reações com pectinas e polifenóis; incorporação de fosfatos inorgânicos ao ATP eADP), e portanto nutricional doSi, ainda não relatada para a mai oria das dicotiledôneas, não acumuladoras do elemento (Emadian \& Newton, 1989; Belanger et al., 1995; Marschner, 1995), como é o caso do eucalipto. Além disso, o período dos experimentos (120 dias) coincidiu com a época em que o eucalipto absorve P mais intensamente (Novais et al., 1990). Portanto, fatores outros que não o Si devem estar também envolvidos na produção de MSPA. (a) $\hat{\mathrm{Y}}=-37,65+0,0055804 * \mathrm{Si}+0,751641 * * \mathrm{E}$ $R^{2}=0,979$

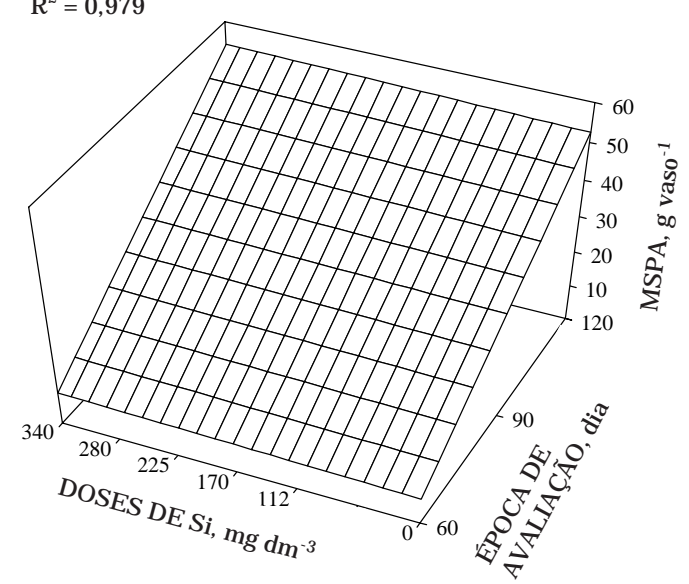

(c) $\hat{\mathrm{Y}}=0,50+0,0356685 * * \mathrm{Si}+0,0001185 * * \mathrm{Si}^{2}+0,300259 * * \mathrm{E}$ $\mathrm{R}^{2}=0,931$

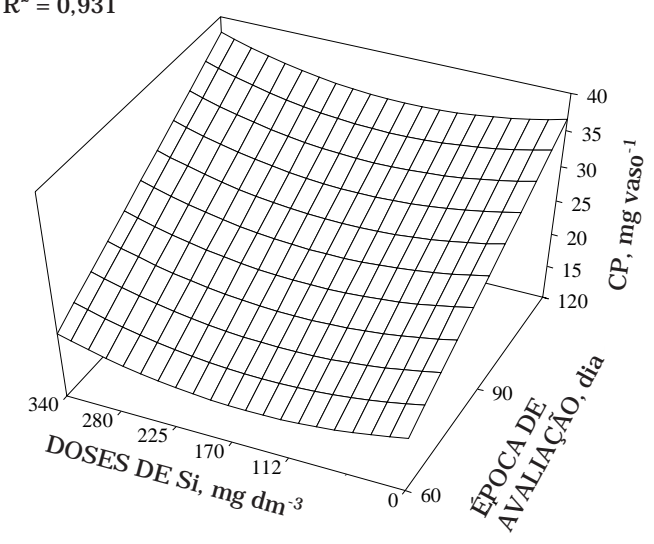

(b) $\hat{\mathrm{Y}}=-29,83+0,0131042 * * \mathrm{Si}+0,533924 * * \mathrm{E}$ $\mathrm{R}^{2}=0,984$

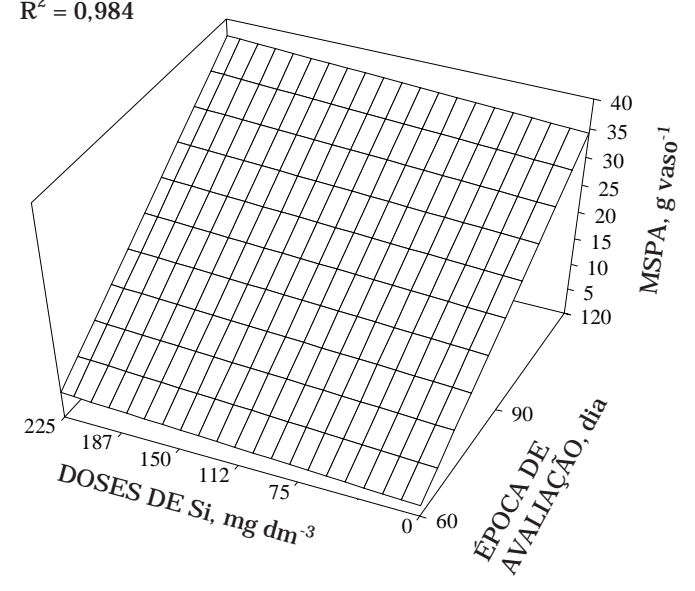

(d) $\hat{\mathrm{Y}}=-48,77+0,0529843^{*} * \mathrm{Si}+0,0001891 * * \mathrm{Si}^{2}+1,28609 * *$ $E-0,0056576 * * E^{2}$
$R^{2}=0,923$

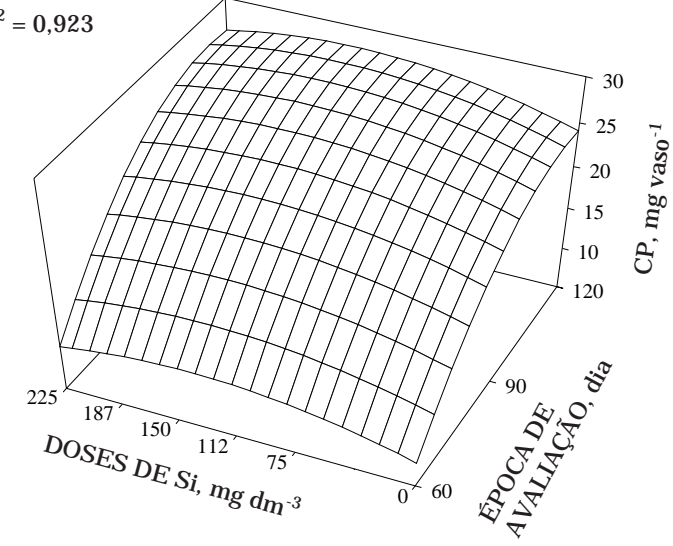

Figura 1. Superfícies de resposta para produção de matéria seca da parte aérea (MSPA) de Eucalyptus grandis cultivado no Latossolo Vermelho-Escuro (a) e Cambissolo (b), e conteúdo de P (CP) na parte aérea das plantas cultivadas no Latossolo Vermelho-E scuro (c) e Cambissolo (d), considerando as doses de $\mathrm{Si}$ e as épocas de avaliação (E ). ** e* significativos a 1 e 5\%, respectivamente. 
Os sinais, negativo do termo "Si" (dose) e positivo do termo "E" (época) do polinômio da figura 1c, indicam que o conteúdo de $P$ (CP) na MSPA das plantas cultivadas no LE diminuiu em função do crescimento das doses de Si no solo e aumentou respondendo a incrementos das épocas de avaliação. Percebe-se, portanto, que o comportamento do CP foi mais bem justificado pelo fator época que pelo fator dose.

O sinal positivo dos termos lineares "Si e E" do polinômio (Figura 1 d) mostra que o CP das plantas cultivadas no Cambissolo aumentou, respondendo a incrementos tanto de doses quanto de épocas. Ao contrário do LE, neste caso, o sinal positivo dos termos lineares, bem como sua idêntica significância (1\%), permiteinferir que não há diferença estatística entre os fatores dose e época em sua capacidade de influenciar o CP das plantas. Pela equação da figura 1d, deduz-se que: (a) o máximo $C P$ (28,03 mg vaso-1 de $\mathrm{P})$ ocorreu aos 113 dias com uma dose de $140 \mathrm{mg} \mathrm{dm}^{-3}$ de Si (ponto máximo obtido da derivação do polinômio); (b) na mesma época, o CP das plantas do tratamento-controle (sem adi ção de $\mathrm{Si}$ ) foi de $24,32 \mathrm{mg} \mathrm{vaso}^{-1}$; (c) o diferencial entre os dois valores, 3,71 mg vaso-1 $^{-1}$, representou um ganho de $15,25 \%$ em $P$. O percentual pode ser considerado de pequena magnitude, quando comparado com os val ores encontrados por Roy et al. (1971), queforam de $47 \%$, em solos cauliníticos, e de $9 \%$, em solos gibbsíticos. Também, no presente trabalho, não se observou aumento no CP das plantas cultivadas no LE, solo que apresenta teores el evados de gibbsita (280 g kg-1, contra $77 \mathrm{~g} \mathrm{~kg}^{-1}$ no solo C). Esseaumento no CP acumulado provavelmente explica o ganho discreto na produção de MSPA das plantas cultivadas no Cambissolo detectado pela superfície de resposta (Figura 1b). Demonstra, também, a sensibilidade do modelo estatístico adotado em explicar o fenômeno atribuindo-o ao fósforo dessorvido por Si.

Hingston et al. (1972) demonstraram, em condições de laboratório com goethita sintética, que a perda do primeiro próton do $\mathrm{H}_{4} \mathrm{SiO}_{4}$ para formar $\mathrm{H}_{3} \mathrm{SiO}_{4}{ }^{-}$(composto silicatado adsorvido no solo após a dessorção do $\mathrm{P}$ previamente retido) só ocorre em $\mathrm{pH} \simeq 9,0$, que coincide com o pk ${ }_{1}$ do $\mathrm{H}_{4} \mathrm{SiO}_{4}$, no entanto, os dados de $\mathrm{pH}$ em água monitorados durante os experimentos (Quadro 2) contrastam com essas observações. Primeiramente, porque, nos dois sol os, esta característica permaneceu constantetanto numa mesma época, apesar do incremento do Si, como ao longo das épocas, não tendo sido detectada significância estatística entre doses de Si, épocas e $\mathrm{pH}$. Em segundo lugar, porque era esperado um aumento de $\mathrm{pH}$, considerando a reconhecida ação alcalinizante da fonte de $\mathrm{Si}$ empregada $\left(\mathrm{CaSiO}_{3}\right)$. Portanto, nestas condições, o mecanismo deadsorção deSi não foi dependentede pH. Defato, McK eague \& Cline (1963b) demonstraram considerável adsorção de Si em solos de Porto Rico (EUA) com pH 4,8.
Quadro 2. Valores do pH em água do Latossolo e do Cambissolo, considerando as doses de Si aplicadas e as épocas de avaliação

\begin{tabular}{|c|c|c|c|}
\hline \multirow{2}{*}{ Dose de Si } & \multicolumn{3}{|c|}{ É poca de avaliação (dia) } \\
\hline & 60 & 90 & 120 \\
\hline$\left(\mathbf{m g ~ d m} \mathbf{~}^{-3}\right)$ & \multicolumn{3}{|c|}{ pH - Latossolo Vermelho-E scuro } \\
\hline 0 & 5,70 & 6,02 & 5,95 \\
\hline 112 & 5,60 & 6,00 & 5,75 \\
\hline 170 & 5,67 & 6,00 & 5,75 \\
\hline 225 & 5,65 & 5,95 & 5,66 \\
\hline 280 & 5,80 & 5,92 & 5,50 \\
\hline \multirow[t]{2}{*}{340} & 5,63 & 5,86 & 5,60 \\
\hline & \multicolumn{3}{|c|}{ pH - Cambissolo } \\
\hline 0 & 4,55 & 5,15 & 5,16 \\
\hline 75 & 4,60 & 5,25 & 5,17 \\
\hline 112 & 4,63 & 5,13 & 5,13 \\
\hline 150 & 4,63 & 5,15 & 5,03 \\
\hline 187 & 4,55 & 5,10 & 5,10 \\
\hline 225 & 4,57 & 5,02 & 5,02 \\
\hline
\end{tabular}

\section{CONCLUSÕES}

1. É possível dessorver fósforo do solo pela ação do silício aplicado como silicato numa etapa posterior à fertilização fosfatada.

2. O ganho em fósforo, de pequena magnitude, foi responsável pelo incrementotambém discreto da produção de matéria seca das plantas cultivadas no Cambissolo.

3. A ação do silício na dessorção de fósforo foi mais evidente no solo menos intemperizado, com maior teor de caulinita (Cambissolo) do que no solo mais intemperizado, com maior teor de gibbsita (Latossolo).

\section{LITE RATURA CITADA}

BALDEON, J .R.M. Efeito da ação al cal inizante e da competição entre silicato e fosfato na eficiência do termofosfato magnesiano em solos ácidos. Piracicaba, Escola Superior de Agricultura "Luiz de Queiroz", 1995. 88 p. (Tese de Doutorado)

BELANGER, R.R.; BOWEN, P.A.; EHRET, D.L. \& MENZIES, J.G. Soluble silicon, its role in the crop and disease management of greenhouse crops. Plant Dis., 74:329-336, 1995.

EMADIAN, S.F. \& NEWTON, R.J . Growth enhancement of loblolly pine (Pinus taeda L.) seedlings by silicon. J . Plant Physiol., 134:98-103, 1989. 
EMPRESA BRASILEIRA DE PESQUISA AGROPECUÁRIA EMBRAPA. Serviço Nacional de Levantamento e Conservação de Solos. Manual de métodos de análise do solo. Rio de J aneiro, 1997. 200p.

HOLT, P.F. \& KING, D.T. Thechemistry of silica surfaces. J . Chem. Soc., 4:773-779, 1955.

HINGSTON, F.J .; POSNER, A.M. \& QUIRK,J .P. Anion adsorption by goethiteand gibbsite. I. The role of the proton in determining adsorption envel opes. J . Soil Sci., 23:177-191, 1972.

KÄMPF, N. \& SCHWERTMANN, U. The 5M NaOH concentration treatment of iron oxides in soils. Clays Clay Miner., 30:401408, 1982.

LEITE, P.C. I nteração silício-fósforo em Latossol o Roxo cultivado com sorgo em casa de vegetação. Viçosa, Universidade Federal de Viçosa, 1997. 87p. (Tese de Doutorado)

MALAVOLTA, E.; VITTI, G.C. \& OLIVEIRA, S.A. Avaliação do estado nutricional das plantas: princípios e aplicações. Piracicaba, POTAFÓS, 1989. 201p.

MARSCHNER, H. Mineral nutrition of higher plants. 2.ed., London, Academic Press, 1995. 889p.

MCKEAGUE,J .A. \& CLINE, M.G. Silica in soil solutions. I. The form and concentration of dissolved silica in aqueous extracts of some soils. Can. J. Soil Sci., 43:70-82, 1963a.

MCKEAGUE, J .A. \& CLINE, M.G. Silica in soil solutions. II. The adsorption of monosilicic acid by soil and by other substances. Can. J . Soil Sci., 43:83-95, 1963b.

MEHRA, O.P. \& J ACKSON, M.L. I ron oxide removal from soils and clays by a dithionite-citrate system buffered with sodium bicarbonate. Clays Clay Miner., 7:317-327, 1960.
NOVAIS, R.F.; BARROS, N.F . \& NEVES, J .C.L. Nutrição mineral do eucalipto. In: BARROS, N.F. \& NOVAIS, R.F., eds. Relação solo-eucalipto. Viçosa, Folha de Viçosa, 1990. p.25-98.

OBIHARA, C.H. \& RUSSEL, E.W. Specific adsorption of the silicate and phosphateby soils. J. Soil Sci., 23:105-117, 1972.

OLIVEIRA, M.G.A. Determinação, adsorção e deslocamento recíprocos de silício e fósforo em Latossolos do Triângulo Mineiro. Viçosa, UniversidadeF ederal deViçosa, 1984. 68p. (Tese de Mestrado)

RESENDE, M.; CURI, N.; RESENDE, S.B. \& CORREA, G.F. Pedologia: base para distinção de ambientes. 2.ed. Viçosa, NEPUT, 1997. 304p.

ROY, A.C.; ALI, M.V.; FOX, R.L. \& SILVA,J .A. Influence of calcium silicate on phosphate solubility and availability in Hawaian Latosols. In: SYMPOSIUM ON SOIL FERTILITY AND EVALUATION, Honolulu, 1971. Proceedings... New Del hi, 1971. p.756-765.

SCHWERTMANN, V. U se of oxalate for Fe extraction from soils. Can. J . Soil Sci., 53:244-246, 1973.

SILVA, J .A. Possible mechanisms for crop response to silicate applications. In: SYMPOSIUM ON SOIL FERTILITY AND EVALUATION, Honolulu, 1971. Proceedings. New Delhi, 1971. p.805-815.

SYERS, J .K.; BROWMAN, M.G.; SAMILLE, G.W. \& COREY, R.B. Phosphate sorption by soils evaluated by the Langmuir adsorption equation. Soil Sci. Soc. Am. Proc., 37:358-363, 1973.

VETTORI, L. Métodos de análise de solos. Rio de J aneiro, Ministério da Agricultura, 1969. 24p. (Boletim técnico, 7) 